\title{
Agregación de sistemas coloidales con cargas opuestas: efecto de la concentración de partículas
}

\author{
A.M. PUERTAS, A. FERNÁNDEZ BARBERO, F.J. DE LAS NIEVES \\ Grupo de Física de Fluidos Complejos. Departamento de Física Aplicada. Universidad de Almería. 04120 Almería.
}

\begin{abstract}
En este trabajo se presentan resultados experimentales de agregación en sistemas mesoscópicos con cargas opuestas. La heteroagregación está controlada por la atracción electrostática. Se ha estudiado el efecto de la proporción relativa de partículas de uno u otro signo y de los potenciales superficiales de ambos sistemas. Las velocidades de agregación más rápidas se han obtenido cuando el sistema más descargado se encuentra en exceso, lo que no puede ser explicado por la teoría cinética habitualmente empleada.
\end{abstract}

Palabras clave: Sistemas mesoscópicos, agregación coloidal, heteroagregación.

Aggregation between oppositely charged colloidal systems: Effect of the relative particle concentration.

In this work we present experimental results on aggregation of mesoscopic systems bearing opposite surface charges. The heteroaggregation is driven by electrostatic attraction. We have studied the effect of the relative particle concentration and of the surface potentials of both systems. The fastest reactions were observed when the discharged system was in excess, which cannot be explained by the kinetic theory usually employed.

Keywords: Mesoscopic systems, colloidal aggregation, heteroaggregation.

\section{INTRODUCCIÓN}

Los sistemas coloidales tienen gran aplicación en diversos procesos industriales. Generalmente, los sistemas que se emplean no se componen de un único tipo de partículas, lo que hace que su comportamiento esté dominado por las interacciones entre partículas de características diferentes. Así, en la recuperación de minerales, la adición de partículas a una pulpa de mineral puede provocar la sedimentación de un determinado tipo de mineral en suspensión o de otro, dependiendo de las características de las partículas que han sido añadidas $(1,2)$.

Si la interacción entre dos tipos de partículas es atractiva o no es suficientemente repulsiva, se producirá agregación. A diferencia de la agregación en un sistema monocomponente (homoagregación), ésta se conoce como heteroagregación. Sin embargo, es necesario notar que en un proceso de heteroagregación, también pueden producirse homoagregaciones de los diferentes sistemas. Dada la complejidad de estas reacciones, el número de trabajos que estudian la heteroagregación desde un punto de vista básico es muy escaso, siendo aún varios aspectos objeto de discusión (3).

El trabajo pionero sobre heteroagregación se debe a Hogg y col. y data de 1966 (4). En él se propone un potencial de interacción para un par de partículas con diferentes potenciales superficiales y radios. Además, se desarrolla un modelo cinético aplicable a los instantes iniciales de la agregación. Tras este trabajo, se han propuesto diferentes potenciales de interacción más exactos que el dado por la aproximación de Hogg y col, aunque éste reproduce las principales características y presenta la ventaja de ser analítico. Sin embargo, la cinética de agregación no ha sido mejorada, y el mismo modelo sigue empleándose.

En este trabajo estudiaremos experimentalmente la agregación en un sistema compuesto por dos tipos de partículas con cargas superficiales de signos opuestos. Al ser sólo dos los componentes del sistema, las posibles reacciones se reducen a tres, lo que simplifica el desarrollo teórico. Además, la interacción entre partículas de cargas opuestas es atractiva, lo que nos permitirá estudiar esta reacción en ausencia de las homoagregaciones de los sistemas. Las agregaciones serán estudiadas mediante dispersión estática de luz, con la que tendremos acceso a los instantes iniciales de forma poco intrusiva.

\section{TEORÍA}

La luz dispersada por una dispersión en la que existen diferentes agregados viene dada por:

$$
I_{T}(k, t)=\sum_{m} I_{m}(k) N_{m}(t)
$$

donde $k$ es el vector de dispersión, que depende del ángulo, $I_{m}$ es la intensidad dispersada por un agregado de $m$ partículas y $N_{m}$ es la concentración de agregados de $m$ partículas. Mediante la aproximación de Rayleigh-Gans-Debye (5), $I_{m}$ 
puede calcularse dependiendo de la estructura interna del agregado. Sin embargo, en la descripción de los instantes iniciales sólo necesitamos los monómeros y dímeros, con lo que simplificaremos el cálculo de la intensidad dispersada, ya que ambos carecen de estructura interna.

Para calcular la evolución temporal inicial de los monómeros y dímeros, recurrimos al modelo desarrollado por Hogg y col. donde se considera agregación irreversible entre partículas de dos tipos:

$$
\begin{aligned}
& \frac{d N_{A}}{d t}=-k_{A A}\left[N_{A}(t)\right]^{2}-k_{A B} N_{A}(t) N_{B}(t) \\
& \frac{d N_{B}}{d t}=-k_{B B}\left[N_{B}(t)\right]^{2}-k_{A B} N_{A}(t) N_{B}(t)
\end{aligned}
$$

$N_{A}$ y $N_{B}$ representan el número de partículas de tipo $A$ y $B$ respectivamente. Las constantes $k_{A A}$ y $k_{B B}$ son las constantes de homoagregación de cada especie mientras que $k_{A B}$ representa la constante de heteroagregación. La evolución del número de monómeros se calculará como:

$$
\begin{gathered}
\frac{d N_{1}}{d t}=\frac{d\left(N_{A}+N_{B}\right)}{d t}=-k_{11}\left[N_{1}(t)\right]^{2} \\
k_{11}=\frac{1}{\left[N_{1}(t)\right]^{2}}\left\{k_{A A}\left[N_{A}(t)\right]^{2}+2 k_{A B} N_{A}(t) N_{B}(t)+k_{B B}\left[N_{B}(t)\right]^{2}\right\}
\end{gathered}
$$

mientras que el de dímeros obedece $N_{1}+N_{2}=N_{0}$, siendo $N_{0}$ el número inicial de partículas. La ecuación anterior puede resolverse si consideramos que en los instantes iniciales los cocientes $N_{A} / N_{1}$ serán prácticamenteconstantes. Introduciendo las soluciones para $N_{1}$ y $N_{2}$ en la ec. [1] tendremos el primer orden en el desarrollo temporal de la intensidad dispersada:

$$
\frac{I_{T}(k, t)}{I_{T}(k, 0)}=(t \rightarrow 0)=1+k_{11} N_{0} \frac{\sin 2 k a}{2 k a} t+O\left(t^{2}\right)
$$

siendo $a$ el radio de la partícula. Por tanto, estudiando la pendiente inicial de la curva de intensidad de luz dispersada en función del tiempo, obtendremos información de $k_{11}$, que nos caracteriza la heteroagregación.

\section{PROCEDIMIENTO EXPERIMENTAL}

Los sistemas experimentales que se han utilizado en este trabajo son dos látex de poliestireno, con grupos superficiales débiles. Ambos sistemas fueron sintetizados en ausencia de emulsificante, y sus principales características se encuentran en la tabla I.

La carga del sistema aniónico proviene de grupos carboxilo, mientras que la del catiónico se debe a grupos amina. Dada la naturaleza débil de ambos grupos, la densidad superficial de carga de estos sistemas será sensible al $\mathrm{pH}$ del medio. En la figura 1 se muestra la variación de la densidad superficial de carga para ambos casos, determinada poten-
TABLA I: CARACTERÍSTICAS DE LOS SISTEMAS COLOIDALES UTILIZADOS.

\begin{tabular}{|c|c|c|c|}
\hline Sistema & Tamaño $^{1}$ & Polidispersidad & Carga máxima $^{2}$ \\
\hline Aniónico & $(183 \pm 7) \mathrm{nm}$ & 1.0039 & $(-22.3 \pm 1.2) \mu \mathrm{C} / \mathrm{cm}^{2}$ \\
\hline Catiónico & $(185 \pm 7) \mathrm{nm}$ & 1.0066 & $(9.2 \pm 2.4) \mu \mathrm{C} / \mathrm{cm}^{2}$ \\
\hline
\end{tabular}

${ }^{1}$ Determinado por microscopía electrónica de transmisión.

2 Obtenido mediante valoración conductimétrica.

TABLA II: CONCENTRACIONES CRÍTICAS DE COAGULACIÓN DE AMBOS SISTEMAS A DIFERENTE PH.

\begin{tabular}{|c|c|c|c|c|}
\hline Sistema & C.C.C. $(\mathrm{pH} 4.5)$ & C.C.C. $(\mathrm{pH} 6)$ & C.C.C. $(\mathrm{pH} 7)$ & C.C.C. (pH 11) \\
\hline Aniónico & $(185 \pm 25) \mathrm{mM}$ & $(470 \pm 24) \mathrm{mM}$ & $(700 \pm 30) \mathrm{mM}$ & $(720 \pm 50) \mathrm{mM}$ \\
\hline Catiónico & $(210 \pm 30) \mathrm{mM}$ & $(211 \pm 15) \mathrm{mM}$ & $(200 \pm 25) \mathrm{mM}$ & $(142 \pm 21) \mathrm{mM}$ \\
\hline
\end{tabular}

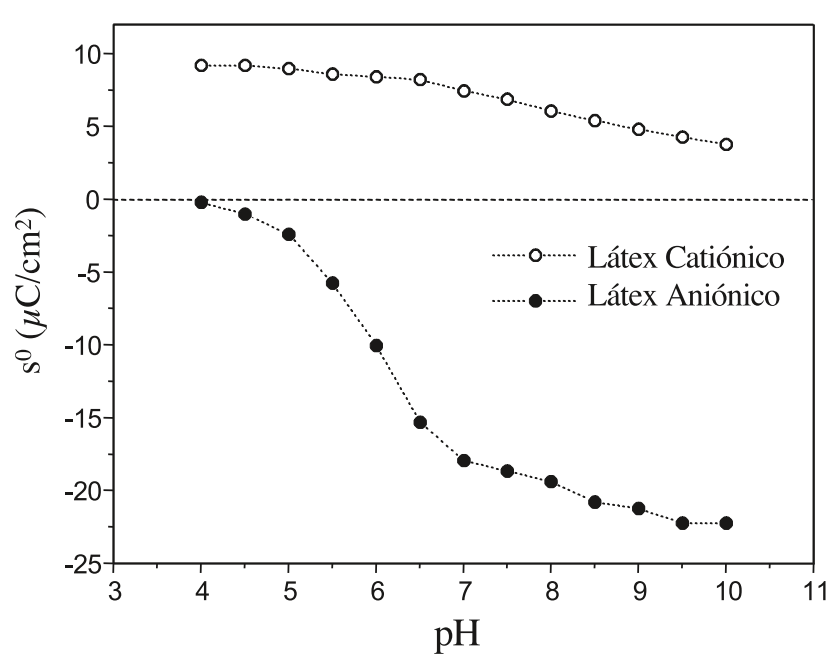

Figura 1. Variación de la carga con el pH para ambos sistemas.

ciométricamente.

Podemos ver en la figura que el sistema aniónico se descarga a $\mathrm{pH} \approx 3.5$ mientras que el catiónico disminuye su carga a $\mathrm{pH}$ básico, aunque no se observa cuando la pierde. Por otra parte, ambos sistemas alcanzan su máxima carga a $\mathrm{pH}^{\prime} \mathrm{s}$ opuestos, como era de esperar.

Esta variación de la carga con el $\mathrm{pH}$ provoca una dependencia de la estabilidad de los sistemas con éste. En la tabla II podemos ver las concentraciones críticas de coagulación (C.C.C.) de ambos sistemas en función del pH. Como cabe esperar, cuanto mayor es la carga de un sistema, mayor es su estabilidad.

\section{RESULTADOS Y DISCUSIÓN}

En esta sección se mostrarán resultados experimentales de la cinética inicial de heteroagregación obtenidos mediante dispersión estática de luz. El dispositivo empleado para este estudio es el modelo comercial de Malvern 4700c. Éste viene diseñado para trabajar en dispersión dinámica de luz, por lo que ha sido necesario aumentar el volumen iluminado para permitir su uso en modo estático.

Las heteroagregaciones se han llevado a cabo a baja concentración de electrolito $([\mathrm{NaCl}]=2.5 \mathrm{mM})$. De esta forma se evita la homoagregación de los sistemas, ya que a tan baja concentración iónica, la carga no ha sido apantallada y las partículas de igual signo de carga se repelen. Por tanto, en la expresión 
de $k_{11}$, ec. [3], podemos tomar $k_{A A}=k_{B B}=0$. Así pues, la dependencia de la pendiente inicial de la curva de intensidad dispersada frente al tiempo en la proporción relativa de partículas se simplifica [6]:

$$
\left.\frac{1}{I_{T}(k, 0)} \frac{d I_{T}(k, t)}{d t}\right|_{t \rightarrow 0}=2 k_{A B} n_{A}\left(1-n_{A}\right) N_{0} \frac{\sin 2 k a}{2 k a}
$$

siendo $n_{A}=N_{A} / N_{0}$. Por tanto, esperamos una dependencia parabólica con $n_{A}$. Por otra parte, el efecto de las cargas superficiales se mostrará únicamente en $k_{A B^{\prime}}$ modificando tan sólo la altura de la parábola.

En la figura 2 se muestra la dependencia experimental con la fracción relativa de partículas negativas de la pendiente inicial de la curva de dispersión de luz frente al tiempo para varios valores del $\mathrm{pH}$. En el rango aquí estudiado el sistema aniónico se descarga, mientras que el catiónico mantiene su carga. Sin embargo, en todos los casos, salvo en el correspondiente a $\mathrm{pH}$ 4, la estabilidad del sistema aniónico es superior a la del catiónico, indicando que su potencial superficial es mayor.

En esta gráfica podemos ver que el resultado esperado no se obtiene. Así, no se observan parábolas en ningún caso, lo que imposibilita el estudio de las curvas en base a la teoría antes expuesta. Además, puede verse que en algunos casos la agregación no llega a producirse, aunque tenemos partículas de signos opuestos en el sistema. La causa de ambos efectos parece encontrarse en la diferencia de potenciales superficiales de los sistemas, ya que conforme ésta disminuye la dependencia se aproxima a la esperada teóricamente.

Podemos incrementar aún más la diferencia de cargas (y por tanto de potenciales superficiales) si descargamos el sistema catiónico, manteniendo el aniónico a su máxima carga. Esta situación se estudia en la figura 3, donde se presentan los datos correspondientes a los valores básicos del $\mathrm{pH}$. Podemos ver que los efectos de asimetría y reacciones paradas que se observaron en la figura 2 aparecen aquí más marcados, lo que de nuevo nos indica que su origen es la diferencia de cargas.

A la vista de estas dos figuras, debemos plantearnos qué está fallando en el modelo que empleamos para describir la heteroagregación. Para resolver esta cuestión es interesante notar que las campanas de velocidad de agregación frente a la fracción relativa de partículas tienen sus máximos cuando la especie más descargada se encuentra en exceso. Por el contrario, cuando ésta se encuentra en minoría, la reacción se ralentiza e incluso llega a no producirse. Si las reacciones que estamos observando se producen entre pares de partículas, el comportamiento debería ser simétrico, por lo que cabe plantearse si la causa de estos efectos se encuentra en reacciones de formación de trímeros (que no son tenidas en cuenta en el modelo).

Si consideramos formación de trímeros, cuando el sistema más cargado se encuentre en exceso, cada dímero (formado por un par de partículas de signos opuestos) se repelerá con el resto de partículas y de dímeros, ralentizando la reacción. Sin embargo, cuando domina el sistema menos cargado, los dímeros atraerán a otras partículas, acelerándose la reacción. Por tanto, es posible que el desacuerdo entre el modelo teórico y los resultados experimentales se deba a la formación de agregados superiores.

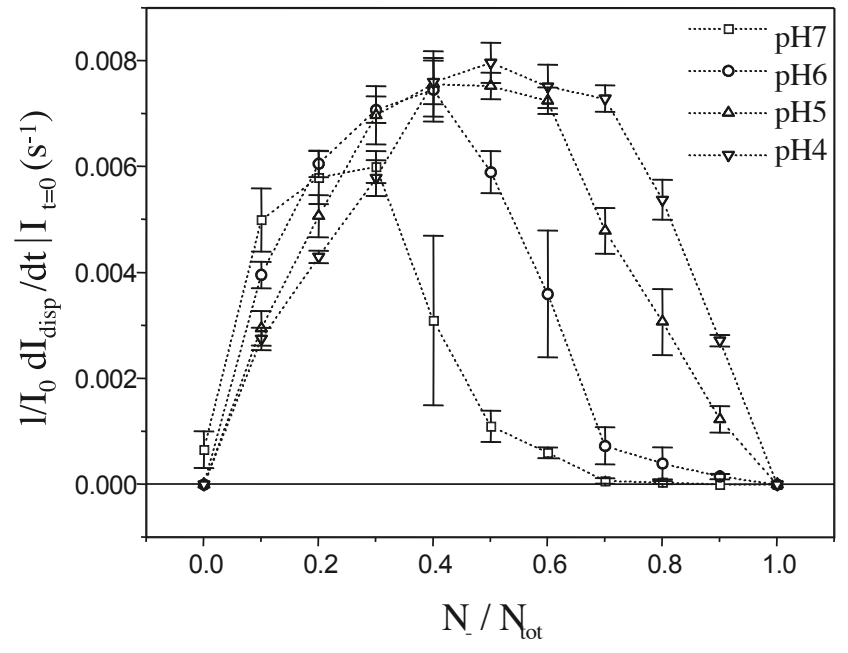

Figura 2. Dependencia experimental de la velocidad inicial de agregación para valores ácidos del $\mathrm{pH}$.

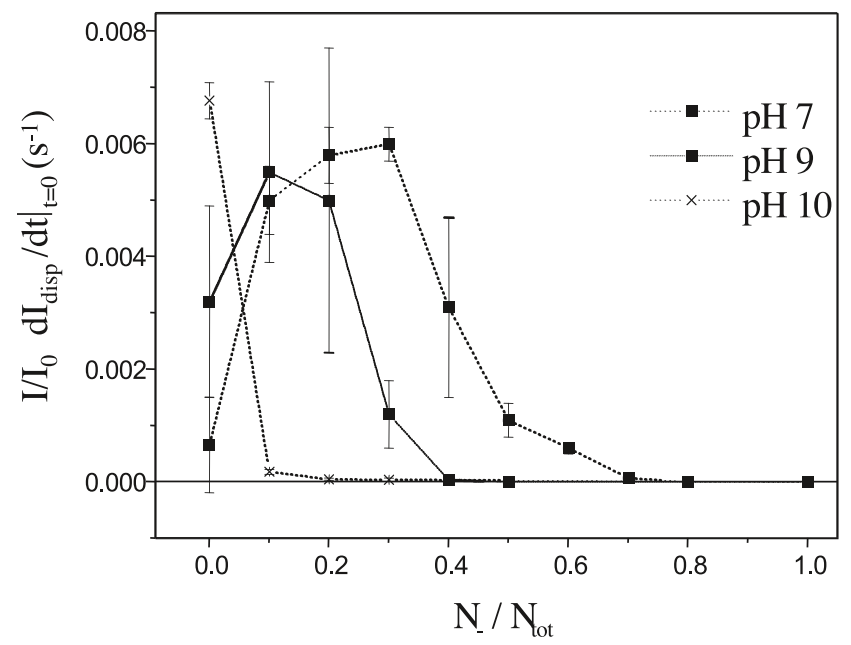

Figura Dependencia experimental de la velocidad inicial de agregación para valores básicos del $\mathrm{pH}$.

Para finalizar, es interesante destacar que se ha mostrado que la reacción de heteroagregación es mucho más compleja que la de homoagregación. Hemos visto cómo la velocidad de agregación es fuertemente dependiente de la fracción relativa de partículas así como de la relación de potenciales superficiales. Las reacciones más rápidas se han obtenido cuando el sistema más descargado se encuentra en exceso.

\section{AGRADECIMIENTOS}

Los autores muestran su agradecimiento al Dr. M.S. Romero Cano por la síntesis de los sistemas coloidales utilizados en este trabajo. Además agradecemos a la CICYT su apoyo económico mediante el proyecto MAT 96-1035-CO3-03. 


\section{BIBLIOGRAFÍA}

1. Q. Wang, K. Heiskanen, Inter. J. Miner. Process. 35121 (1992).

2. Q. Wang, K. Heiskanen, Inter. J. Miner. Process. 35133 (1992).

3. A.M. Puertas, J.A. Maroto, A. Fernández Barbero, F.J. de las Nieves, Phys. Rev. E 591943 (1999)
4. R. Hogg, T.W. Healy, D.W. Fuerstenau, Trans. Faraday Soc. 621638 (1966).

5. M. Kerker, The Scattering of Light and Other Electromagnetic Radiation Academic Press, Nueva York, 1969

6. N. Ryde, E. Matijevic, J. Chem. Soc. Faraday Trans. I 90167 (1994). 